\title{
AGXT2L1 is downregulated in carcinomas of the digestive system
}

\author{
YUNCHAO DENG ${ }^{1,2^{*}}$, LU WU $^{1,2^{*}}$, QIANSHAN DING ${ }^{1,2}$ and HONGGANG $\mathrm{YU}^{1,2}$ \\ ${ }^{1}$ Department of Gastroenterology, ${ }^{2}$ Hubei Key Laboratory of Digestive System, \\ Renmin Hospital of Wuhan University, Wuhan, Hubei 430060, P.R. China
}

Received April 24, 2019; Accepted January 16, 2020

DOI: $10.3892 / \mathrm{ol} .2020 .11645$

\begin{abstract}
Alanine-glyoxylate aminotransferase 2-like 1 (AGXT2L1) is a modulator of phospholipid metabolism, and its role in tumor biology is obscure. Previously, significant downregulation of AGXT2L1 has been observed in hepatocellular carcinoma. The aim of the present study was to investigate AGXT2L1 expression and its association with the clinical characteristics of common carcinomas of the digestive system. In the present study, the expression levels of AGXT2L1 were detected by immunohistochemical staining in colorectal cancer (CRC), gastric cancer and pancreatic cancer tissues. The associations between AGXT2L1 expression and clinicopathological features were analyzed using public gene expression datasets. Small interfering RNA was transfected into SW480 and HCT116 cells to explore the role of AGXT2L1 in CRC cells. AGXT2L1 expression was significantly decreased in cancerous tissues compared with in normal tissues, and low AGXT2L1 expression was associated with an unfavorable prognosis in patients. Furthermore, it was revealed that AGXT2L1 may regulate phosphatidylinositol and phosphatidylserine metabolism in cancerous tissues, and that decreased AGXT2L1 expression could induce autophagy in CRC cells. Overall, the present study provides a basis for further understanding of the role of AGXT2L1 and its association with autophagy in cancer.
\end{abstract}

\section{Introduction}

Carcinoma of the digestive system, including hepatocellular carcinoma (HCC), colorectal cancer (CRC), gastric cancer (GC) and pancreatic cancer (PC), is one of the leading causes of death worldwide (1). Although the molecular mechanisms

Correspondence to: Dr Honggang Yu or Dr Qianshan Ding, Department of Gastroenterology, Renmin Hospital of Wuhan University, 238 Jiefang Road, Wuhan, Hubei 430060, P.R. China

E-mail: yuhonggang1968@163.com

E-mail: iamdqs@163.com

${ }^{*}$ Contributed equally

Key words: alanine-glyoxylate aminotransferase 2-like 1, digestive cancer, pathology, autophagy and genetic changes underlying these cancer types have been gradually unveiled in recent years, there remain obstacles in the treatment of these diseases, due to complexity and heterogeneity in molecular pathogenesis. Therefore, it is necessary to discover and develop more effective diagnostic biomarkers and potential therapeutic targets for these diseases.

Alanine-glyoxylate aminotransferase 2-like 1 (AGXT2L1) was initially discovered in brain tissues, and is considered to be involved in the pathogenesis of schizophrenia and bipolar disorder (2-4). Although AGXT2L1 belongs to a subfamily of class II aminotransferases, its enzyme activity is much stronger as a phosphorylase than as an aminotransferase (2). AGXT2L1 is identified to be a phosphoethanolamine (PEtN) phosphorylase and can irreversibly degrade PEtN to acetaldehyde, phosphate and ammonia. In a previous study, it has been discovered that AGXT2L1 is significantly decreased and potentially functions as a tumor suppressor in $\mathrm{HCC}$, and it may regulate the de novo lipogenesis of cancer cells (5). That study was the first to investigate the expression and function of AGXT2L1 in cancer. However, its role remains obscure in other types of cancer. In the present study, the aim was to detect the expression levels of AGXT2L1 in carcinomas of the digestive system and determine its biological functions in different types of digestive cancer.

Autophagy is a process in which cells degrade organelles and proteins to accomplish both the self-maintenance of cells and the recycling of intracellular substances $(6,7)$. In the processes of tumorigenesis and cancer progression, autophagy may be activated to degrade unnecessary macromolecules to meet the increased proliferation requirements $(6,7)$. $\mathrm{PEtN}$ is a precursor of phosphatidylethanolamine (PE) (8), which is the main target for GABA type A receptor associated protein like 2 (ATG8) conjugation during autophagosome formation (9). Therefore, it was hypothesized that AGXT2L1 may be involved in a regulatory mechanism of autophagy. Among the proteins involved in autophagy, microtubule-associated protein 1 light chain 3 is the most extensively used autophagy marker, which undergoes lipidation with PE following autophagy induction, resulting in the formation of the phagophore membrane-bound form, LC3-II (10). LC3 expression was detected via western blotting and immunofluorescence, in order to determine autophagy activity. Gene set enrichment analysis (GSEA), combined with in vitro experiments were performed in the present study, in order to determine the role AGXT2L1 plays in colon cancer.

Together with a previous study on HCC, the present study demonstrated that AGXT2L1 is downregulated in carcinomas 
of the digestive system and may function as a promising diagnostic and prognostic biomarker for these diseases.

\section{Materials and methods}

Clinical samples. Primary gastric cancer and CRC mucosal tissues and corresponding adjacent normal mucosal tissues (5-10 cm away from tumor) were obtained from 16 patients with GC (10 men and 6 women; age range, 47-71 years; mean age, 58.8 years) and six patients with CRC ( 3 men and 3 women; age range, 47-64 years; mean age, 56.2 years) undergoing surgical resection at Renmin Hospital of Wuhan University (Wuhan, China) between October 2016 and January 2017. All patients had never received chemotherapy or radiotherapy. Written informed consent was obtained from each patient. All tissues were snap-frozen in liquid nitrogen and stored at $-80^{\circ} \mathrm{C}$. All samples were validated by two experienced histopathologists as tumor or normal tissues.

Cell culture, culture conditions and antibodies. The human colon cancer HCT116 and SW480 cell lines were cultured in DMEM (Hyclone; GE Healthcare Life Science) supplemented with $10 \% \mathrm{FBS}, 100 \mathrm{U} / \mathrm{ml}$ penicillin and $100 \mathrm{mg} / \mathrm{ml}$ streptomycin (Hyclone; GE Healthcare Life Science) at $37^{\circ} \mathrm{C}$ with $5 \% \mathrm{CO}_{2}$. A rabbit polyclonal anti-AGXT2L1 antibody was obtained from Sigma-Aldrich (Merck KGaA; cat. no. HPA044546). A mouse monoclonal anti-GAPDH antibody was obtained from Santa Cruz Biotechnology, Inc. (cat. no. sc-47724). A rabbit anti-LC3B antibody was obtained from Cell Signaling Technology, Inc. (cat. no. 2775S). Horseradish peroxidase (HRP)-labeled goat anti-rabbit immunoglobulin G ( $\mathrm{IgG})(\mathrm{H}+\mathrm{L})$, HRP-labeled goat anti-mouse IgG $(\mathrm{H}+\mathrm{L})$ and FITC-labeled goat anti-rabbit IgG $(\mathrm{H}+\mathrm{L})$ antibodies were obtained from Beyotime Institute of Biotechnology (cat. no. A0208, A0216 and A0562, respectively).

Tissue sampling and immunohistochemistry staining. GC, $\mathrm{CRC}, \mathrm{PC}$ and esophageal cancer tissues from patients were retrieved from the Department of Pathology at Renmin Hospital of Wuhan University, fixed in $10 \%$ neutral buffered formalin fixation (Beijing Solarbio Science \& Technology Co., Ltd.) for $24 \mathrm{~h}$ at room temperature and embedded in paraffin. Paraffin-embedded tissue samples were cut into 5- $\mu$ m-thick sections and subsequently deparaffinized in xylene for $15 \mathrm{~min}$ at room temperature and rehydrated in a descending ethanol series $(100,95,70$ and $50 \%$ for $5 \mathrm{~min}$ at room temperature, respectively). Deparaffinized sections were incubated with in $0.1 \mathrm{M}$ citric acid buffer ( $\mathrm{pH}$ 6.0; www.servicebio.cn), prior to incubation with $0.3 \%$ hydrogen peroxide (in PBS) for $10 \mathrm{~min}$ at room temperature to inhibit endogenous peroxidase activity. Sections were subsequently blocked with 5\% BSA (provided in the ready-to-use IHC kit; cat. no. KIT-9710; Fuzhou Maxim Biotech. Co., Ltd..) at room temperature for $15 \mathrm{~min}$. Tissue sections were incubated with primary antibody directed against AGXT2L1 (1:200; cat. no. HPA044546; Merck KGaA) overnight at $4^{\circ} \mathrm{C}$. Following the primary incubation, sections were incubated with a goat anti-rabbit $\operatorname{IgG}(\mathrm{H} \& \mathrm{~L})$ biotin-conjugated secondary antibody (provided in the ready-to-use IHC kit; cat. no. KIT-9710; Fuzhou Maxim Biotech. Co., Ltd) for $15 \mathrm{~min}$ at room temperature. An UltraSensitive ${ }^{\mathrm{TM}} \mathrm{SP}$ (mouse/rabbit) Immunohistochemistry kit (Fuzhou Maxim
Biotech. Co., Ltd.) and a 3,3'-diaminobenzidine (DAB) kit (cat. no. DAB-0031; Fuzhou Maxim Biotech Co., Ltd.) were used for immunohistochemistry. The slides were incubated at room temperature in alkaline phosphatase-conjugated streptavidin for $30 \mathrm{~min}$ followed by DAB solution. The chromogenesis procedure was monitored under a light microscope (BX43 Upright Microscope; magnification, x100; Olympus Corporation). The intensity and percentage of stained sections were independently assessed by two certified pathologists from the Department of Pathology, Renmin Hospital of Wuhan University (Wuhan, China) and verified using ImageJ software (version 1.51j8, National Institutes of Health).

Gene expression profile analysis. The gene expression profile GSE17536 (11) was downloaded from the National Center for Biotechnology Information Gene Expression Omnibus database (https://www.ncbi.nlm.nih.gov/geo). This dataset contains general information regarding patients with CRC, such as age, sex, tumor stage, tumor grade and survival data. The patients were divided into two groups according to their AGXT2L1 expression level (top 50\%, high; vs. bottom 50\%, low) for the receiver operating characteristic (ROC) curve analysis. ROC analysis was performed to determine the optimal cut-off value for the CRC survival group, as determined using Youden's index, in order to screen AGXT2L1 expression. Based on this analysis, patients were grouped into high and low expression subgroups. Subsequently, the association between AGXT2L1 and the clinical features of patients was analyzed. The GSE63089 (12) and GSE27342 $(13,14)$ datasets were downloaded from the National Center for Biotechnology Information Gene Expression Omnibus database, in order to assess AGXT2L1 expression in gastric cancer. For GSEA analysis, GSEA tools (http://www.broadinstitute.org/gsea) and the gene expression profile from the GSE17536 dataset were used to analyze the potential genes influenced by AGXT2L1, as previously described (5).

Small interfering (si)RNA transfection. siRNAs were designed as previously described (5), and were obtained from Shanghai GenePharma Co., Ltd. At 24 h before transfection, HCT116 and SW480 cells were seeded into 6-well plates at a density of $1 \times 10^{6}$ cells/well. Once cells reached $30-50 \%$ confluence, Lipofectamine $^{\circledR} 2000$ (Invitrogen; Thermo Fisher Scientific, Inc.) was used to transfect siRNAs $(100 \mathrm{pmol})$ into cells. At 3 days following transfection, cells were collected and protein was extracted. The most efficient siRNA was selected for the subsequent experiments. The sequences used were as follows: AGXT2L1 forward, 5'-CCGGAGAAACUCUCUGUUUTT-3' and reverse, 5'-AAACAGAGAGUUUCUCCGGTT-3'; and non-targeting siRNA forward, 5'-UUCUCCGAACGUGUCA CGUTT-3' and reverse, 5'-ACGUGACACGUUCGGAGA ATT-3'.

Western blotting. Total protein was extracted from cells transfected with siRNA and pre-frozen tissue samples using RIPA lysis buffer supplemented with $1 \mathrm{mM}$ PMSF (Beyotime Institute of Biotechnology). Lysates were centrifuged at $13800 \mathrm{x} \mathrm{g}$ for $15 \mathrm{~min}$ at $4^{\circ} \mathrm{C}$ and the supernatant was used for subsequent analysis. Total protein was quantified using a bicinchoninic acid assay and $30 \mu \mathrm{g}$ protein/lane was 

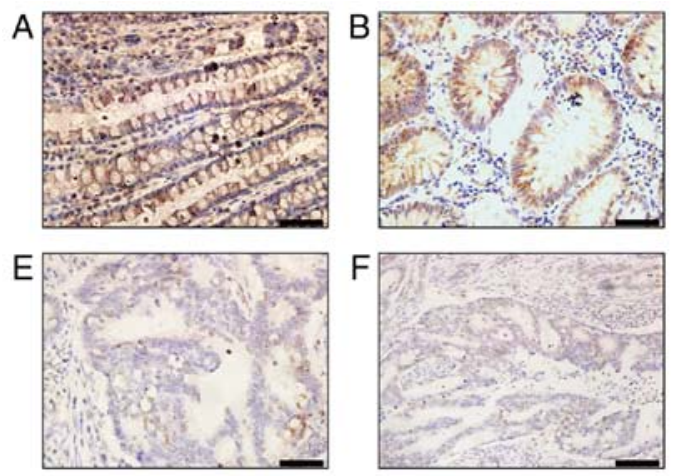
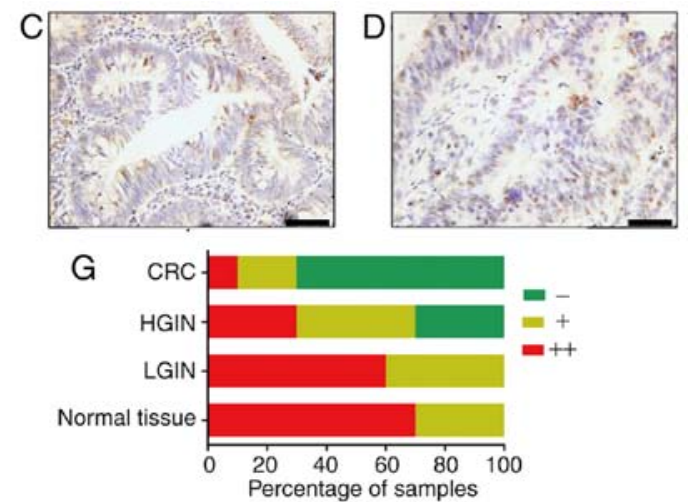

Figure 1. AGXT2L1 expression is decreased in CRC tissues. (A) Representative strong AGXT2L1 expression (++) in normal colonic tissue. (B) Representative AGXT2L1 expression (++) in LGIN tissues. (C) Representative AGXT2L1 expression (+) in HGIN tissues. (D-F) Representative AGXT2L1 expression (-) in CRC tissues. (D) Well differentiated; (E) moderately differentiated; (F) poorly differentiated. (G) AGXT2L1 expressed as a percentage of cases in normal colon tissues, intraepithelial neoplasia tissues and CRC tissues. Scale bars, $50 \mu \mathrm{m}$. AGXT2L1, alanine-glyoxylate aminotransferase 2-like 1; CRC, colorectal cancer; HGIN, high-grade colonic intraepithelial neoplasia; LGIN, low-grade colonic intraepithelial neoplasia.

separated via SDS-PAGE on a $10 \%$ polyacrylamide gel. The separated proteins were subsequently transferred onto a polyvinylidene difluoride membrane (Merck KGaA) and blocked with $5 \%$ non-fat milk in TBS with $0.1 \%$ Tween-20 for $1 \mathrm{~h}$ at room temperature. Membranes were washed three times in TBST buffer ( $5 \mathrm{~min} /$ wash) and incubated with primary antibodies against AGXT2L1 (1:1,000; cat. no. HPA044546; Merck KGaA), LC3B (1:1,000; cat. no. 2775S; Cell Signaling Technology, Inc.) and GAPDH (1:400; cat. no. sc-47724; Santa Cruz Biotechnology, Inc.) on a shaker, overnight at $4^{\circ} \mathrm{C}$. Following the primary incubation, membranes were incubated with horseradish peroxidase (HRP)-labeled goat anti-rabbit immunoglobulin $\mathrm{G}(\mathrm{IgG})(\mathrm{H}+\mathrm{L})$ (cat. no. A0208) and HRP-labeled goat anti-mouse IgG $(\mathrm{H}+\mathrm{L})$ (cat. no. A0216) for $2 \mathrm{~h}$ at room temperature (both 1:1,000 and both from Beyotime Institute of Biotechnology). Membranes were washed three times with TBS, with $0.1 \%$ Tween-20 and protein bands were visualized using ECL reagents (Wuhan Servicebio Technology Co., Ltd.). Signals were detected using the Gel Doc XR system (Bio-Rad Laboratories, Inc.) and band intensity was measured via densitometry using Image Lab software (version 5.1, Bio-Rad Laboratories, Inc.).

RNA extraction and reverse transcription-quantitative (RT- $q)$ $P C R$. Total RNA was extracted from tissue samples using TRIzol (Invitrogen; Thermo Fisher Scientific, Inc.), according to the manufacturer's protocol. cDNA synthesis was performed using a PrimeScript ${ }^{\mathrm{TM}}$ RT reagent kit with gDNA Eraser (cat. no. RR047A; Takara Bio, Inc.). The RT reaction conditions were as follows: $37^{\circ} \mathrm{C}$ for $15 \mathrm{~min}$, followed by $85^{\circ} \mathrm{C}$ for 5 sec. mRNA levels were quantified with a Real-Time PCR system (Applied Biosystems; Thermo Fisher Scientific, Inc.) using SYBR Premix EX Taq II (RR8201; Takara Bio, Inc.). The PCR conditions were as follows: Initial denaturation at $95^{\circ} \mathrm{C}$ for $30 \mathrm{sec} ; 38$ cycles of denaturation at $95^{\circ} \mathrm{C}$ for $5 \mathrm{sec}$ and annealing/extension at $60^{\circ} \mathrm{C}$ for $34 \mathrm{sec}$. GAPDH was used as an internal control. The primers were, AGXT2L1 forward, 5'-GCCGATGGACCTCATAGAAA-3' and reverse, 5'-TGG GCTTCTTTCAGCATCTT-3'; GAPDH forward, 5'-CAAGG CCAACCGCGAGAA-3' and reverse, 5'-CCCTCGTAGA TGGGCACAGT-3'. The expression of AGXT2L1 relative to
GAPDH was determined using the $2^{-\Delta \Delta \mathrm{Cq}}$ method (15). The reactions were repeated three times.

Immunofluorescence detection. Cells were seeded onto coverslips in 24-well plates, at a density of $1 \times 10^{4}$ and cultured at $37^{\circ} \mathrm{C}$, in $5 \% \mathrm{CO}_{2}$ and $95 \%$ humidified air overnight. The next day, cells were transfected with siRNA. After $24 \mathrm{~h}$, the cells were washed with PBS three times. Subsequently, $4 \%$ paraformaldehyde was used to fix the cells for $15 \mathrm{~min}$ at room temperature. Next, cells were permeabilized in $0.5 \%$ Triton X-100 for $10 \mathrm{~min}$ at room temperature. Following that, cells were blocked in 5\% BSA for $30 \mathrm{~min}$ at room temperature and incubated with the primary antibody against LC3B (1:400; cat. no. 2775S; Cell Signaling Technology, Inc.) overnight at $4^{\circ} \mathrm{C}$, followed by incubation with secondary antibodies at room temperature for $1 \mathrm{~h}$. After the addition of the FITC-labeled goat anti-rabbit IgG $(\mathrm{H}+\mathrm{L})$ secondary antibody (1:400; cat. no. A0562; Beyotime Institute of Biotechnology), all steps were performed in the dark. Images were captured under a fluorescence microscope (Olympus Corporation).

Statistical analysis. Statistical analysis was performed using SPSS software (version 20.0; IBM Corp.) and GraphPad Prism software (version 6.0.1; GraphPad Software, Inc.). The Kaplan-Meier method and the log-rank test were used to analyze the associations between AGXT2L1 expression and the survival of patients with CRC. The associations between AGXT2L1 expression and clinicopathological features were statistically analyzed by the $\chi^{2}$ test. Other data were statistically analyzed using paired Student's t-test. All data are presented as the mean \pm standard deviation. All experiments were performed in triplicate. $\mathrm{P}<0.05$ was considered to indicate a statistically significant difference.

\section{Results}

AGXT2L1 expression is decreased in CRC. To explore the expression levels of AGXT2L1 in CRC, normal, intraepithelial neoplasia and CRC tissues were collected. Subsequently, AGXT2L1 expression was detected by immunohistochemistry. Most normal tissues (Fig. 1A) and colonic low-grade 

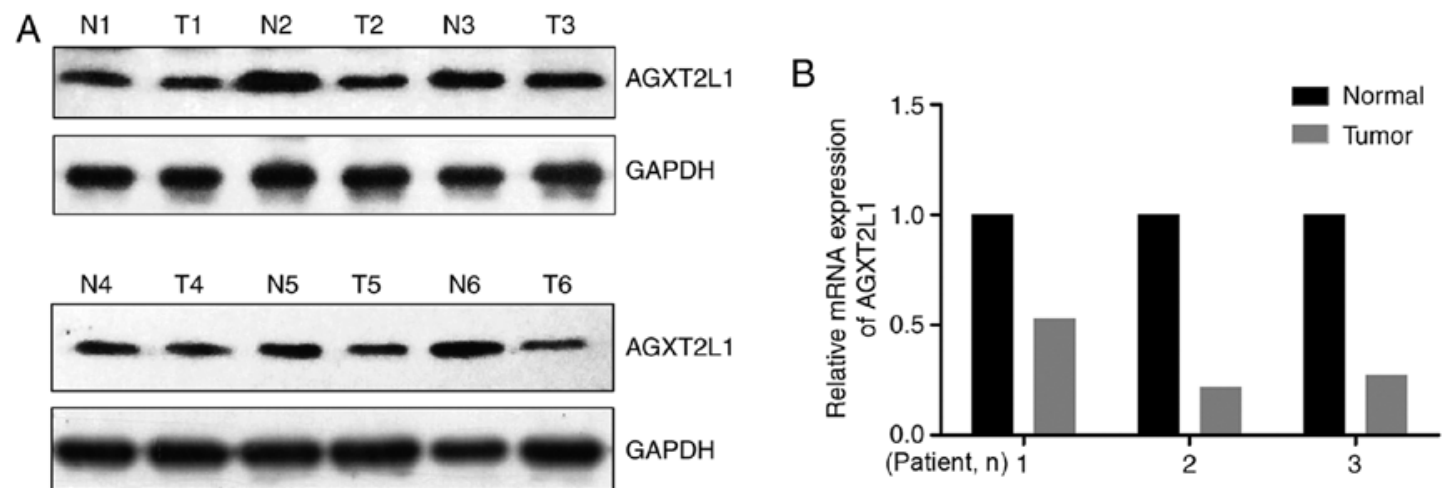

Figure 2. AGXT2L1 expression is reduced at the protein and mRNA levels in patients with CRC. (A) AGXT2L1 protein expression was lower in CRC tissues compared with in matched normal tissue. (B) AGXT2L1 mRNA expression was notably reduced in 3 cases of CRC compared with in matched normal tissue. CRC, colorectal cancer; AGXT2L1, alanine-glyoxylate aminotransferase 2-like 1; N, normal; T, tumor.

Table I. Association between AGXT2L1 expression and clinicopathological features in patients with colorectal cancer (GSE17536).

\begin{tabular}{lrrrr}
\hline & \multicolumn{4}{c}{ AGXT2L1 expression } \\
\cline { 2 - 5 } Characteristics & Patients, $\mathrm{n}$ & Low, $\mathrm{n}$ & High, $\mathrm{n}$ & P-value \\
\hline Age, years & & & & 0.045 \\
$\leq 65$ & 78 & 69 & 9 & \\
$>65$ & 99 & 76 & 23 & \\
Sex & & & & 0.801 \\
Male & 96 & 78 & 18 & \\
Female & 81 & 67 & 14 & \\
AJCC stage & & & & 0.027 \\
T1 & 24 & 16 & 8 & \\
T2 & 57 & 43 & 14 & \\
T3 & 57 & 51 & 6 & \\
T4 & 39 & 35 & 4 & \\
Grade & & & & 0.592 \\
WD & 16 & 13 & 3 & \\
MD & 134 & 108 & 26 & \\
PD & 27 & 24 & 3 & \\
\hline
\end{tabular}

WD, well differentiated; MD, moderately differentiated; PD, poorly differentiated; AJCC, American Joint Committee on Cancer; AGXT2L1, alanine-glyoxylate aminotransferase 2-like 1 .

intraepithelial neoplasia (LGIN) tissues (Fig. 1B) were strongly AGXT2L1-positive (70\%, 7/10; 60\%, 6/10, respectively), and a small number of tissues were weakly positive $(30 \%, 3 / 10$; $40 \%, 4 / 10$, respectively). In colonic high-grade intraepithelial neoplasia (HGIN) tissues and CRC tissues, most samples were AGXT2L1-negative or weakly positive. In colonic HGIN (Fig. 1C), 30\% (3/10) of the tissues were AGXT2L1 negative, $40 \%(4 / 10)$ were weakly positive and $30 \%$ (3/10) were positive; in CRC tissues (Fig. 1D-F), 70\% (7/10) of the tissues were AGXT2L1 negative, 20\% (2/10) were weakly positive and only $10 \%(1 / 10)$ were strongly positive. Notably, it was observed that AGXT2L1 expression decreased gradually from normal tissue to intraepithelial neoplasia to colon cancer (Fig. 1G), suggesting that AGXT2L1 serves an important role in the malignant transformation of colonic tissue. The expression levels of AGXT2L1 were also detected in six pairs of CRC tissues and corresponding adjacent tissues by western blotting. It was demonstrated that the expression levels of AGXT2L1 in CRC tissues were lower compared with those in the adjacent tissues (Fig. 2A). Additionally, it was identified that the mRNA expression levels of AGXT2L1 were decreased in three CRC tissues compared with in paired adjacent tissues (Fig. 2B). Collectively, these results indicated that AGXT2L1 was downregulated in CRC.

Clinicopathological features and the survival of patients with CRC are associated with AGXT2L1 expression. A total of 177 patients with CRC in the GES17536 dataset were divided into two groups (high expression: $\geq 4.8355, \mathrm{n}=32$; low expression: $<4.8355, \mathrm{n}=145$ ). The association between the clinicopathological features and AGXT2L1 expression was analyzed statistically using the $\chi^{2}$ test. AGXT2L1 expression was closely associated with age $(\mathrm{P}=0.045)$ and American Joint Committee on Cancer $\mathrm{T}$ stage $(\mathrm{P}=0.027$; Table I). Survival analysis was subsequently performed using the Kaplan-Meier method to estimate the association between AGXT2L1 expression and the survival of patients with CRC. It was demonstrated that AGXT2L1 expression was significantly associated with the prognosis of patients with CRC (Fig. 3A); patients with high expression levels of AGXT2L1 displayed longer overall survival $(\mathrm{P}=0.036)$ and possibly suffered less incidence of recurrence of the disease $(\mathrm{P}=0.073)$ compared with patients with low expression levels of AGXT2L1 (Fig. 3B).

AGXT2L1 expression is also decreased in GC and $P C$. Furthermore, the expression levels of AGXT2L1 were examined in gastric tissues. Similar to the expression in colonic tissues, AGXT2L1 expression also decreased gradually from normal gastric tissue to intraepithelial neoplasia to GC (Fig. 4). In normal gastric tissues (Fig. 4A), 90\% (9/10) of the samples were strongly positive and $10 \%(1 / 10)$ of the samples were weakly positive, whereas in LGIN tissues (Fig. 4B), 50\% (5/10) of the samples were strongly positive and $50 \%(5 / 10)$ of the samples were weakly positive. In HGIN tissues (Fig. 4C), 20\% (2/10) 

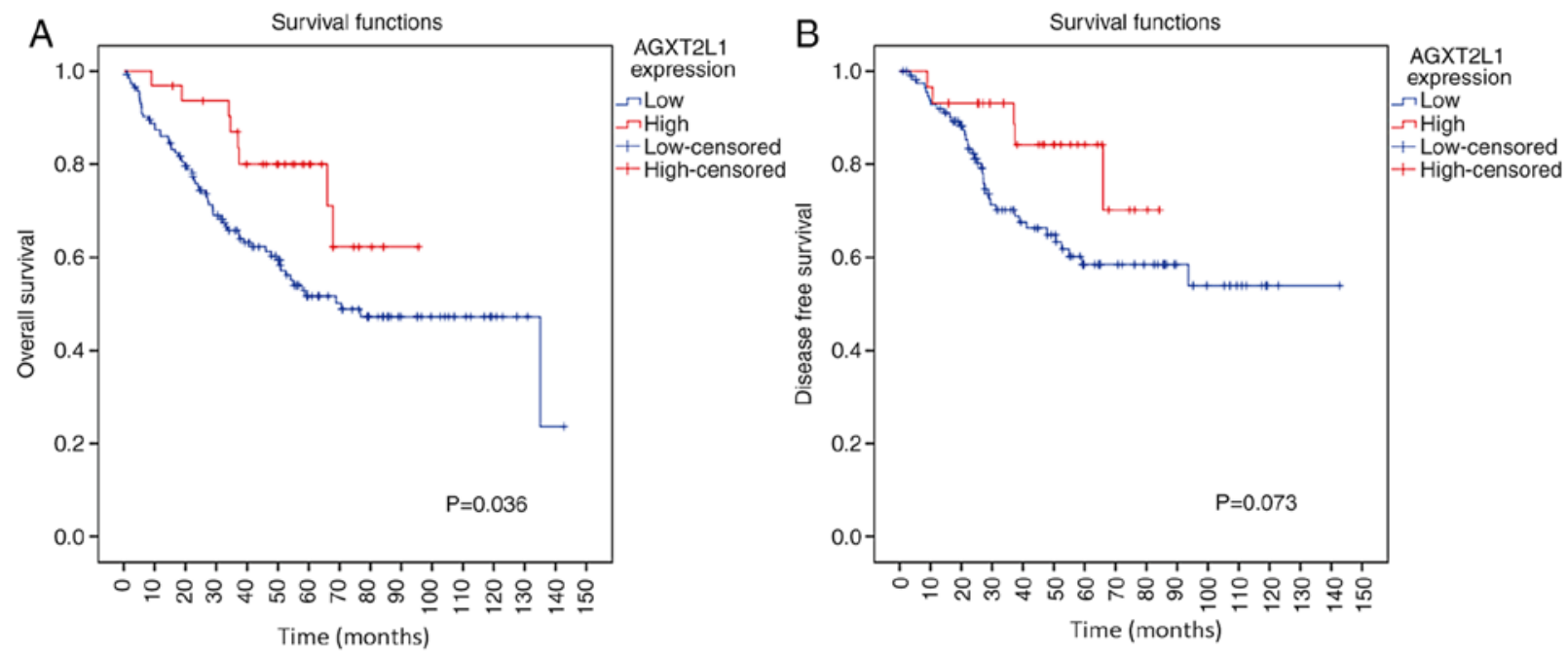

Figure 3. AGXT2L1 expression is associated with the survival of patients with colorectal cancer. (A) High expression of AGXT2L1 indicated a high overall survival rate. (B) AGXT2L1 expression was not significantly associated with disease-free survival. P-values were derived from a log-rank test. AGXT2L1, alanine-glyoxylate aminotransferase 2-like 1 .
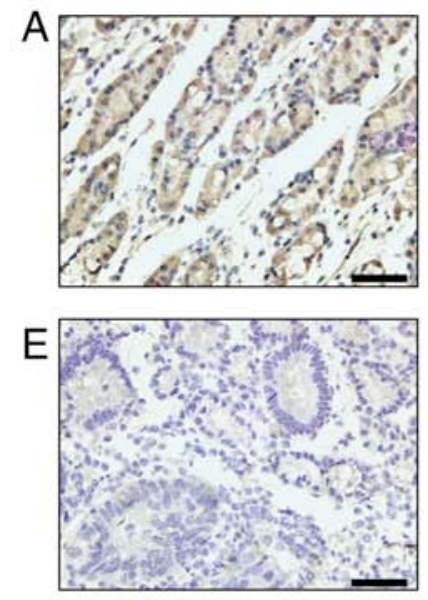

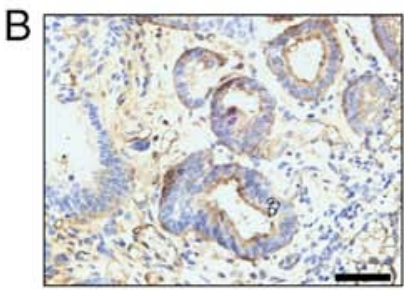

$\mathrm{F}$

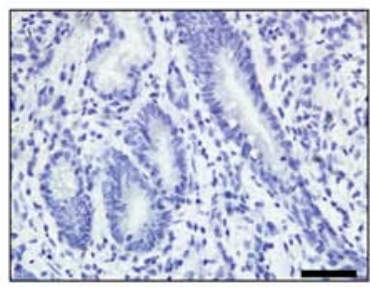

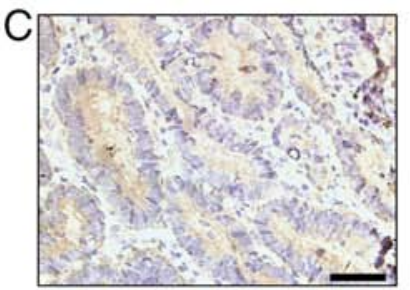
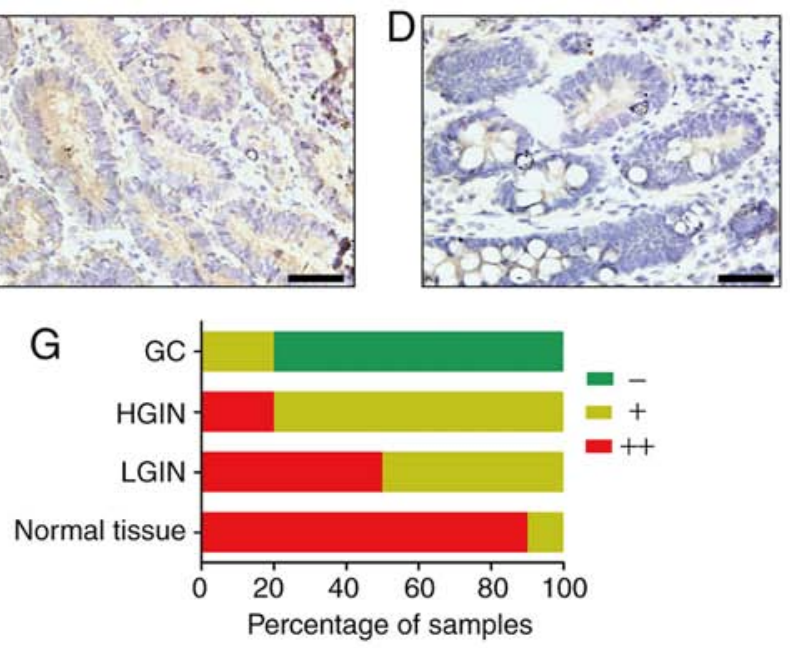

Figure 4. AGXT2L1 expression is decreased in GC tissues. AGXT2L1 expression in gastric tissues was determined by immunohistochemistry. Representative images of (A) normal gastric tissue, (B) LGIN and (C) HGIN, and (D) well-differentiated, (E) poorly differentiated and (F) undifferentiated GC tissues are shown. (G) Percentage of samples at each expression level in normal, LGIN, HGIN and GC tissues. Scale bars, $50 \mu \mathrm{m}$. LGIN, low-grade intraepithelial neoplasia; HGIN, high-grade intraepithelial neoplasia; GC, gastric cancer; AGXT2L1, alanine-glyoxylate aminotransferase 2-like 1.

of the samples were strongly positive and $80 \%(8 / 10)$ of the samples were weakly positive. The absence of AGXT2L1 was detected only in GC tissues (80\%; 16/20; Fig. 4D-F). Analysis of the public datasets GSE63089 (Fig. 5A) and GSE27342 (Fig. 5B), and the detection of AGXT2L1 by western blotting (Fig. 5C) and RT-qPCR (Fig. 5D) also demonstrated that AGXT2L1 was downregulated in GC. Subsequently, AGXT2L1 was detected in four pairs of PC tissues and their corresponding adjacent tissues. All normal tissues (Fig. 6A) exhibited high expression levels of AGXT2L1, whereas all cancerous tissues (Fig. 6B) displayed low expression or absence of AGXT2L1. AGXT2L1 expression was examined in esophageal cancer tissues by immunohistochemistry, however, AGXT2L1 intensity was weak or absent in esophagus tissues (data not shown), indicating that AGXT2L1 expression may be cell-type specific.
AGXT2L1 is associated with PI metabolism and is involved in autophagy. Gene Set Enrichment Analysis using the CRC expression profile data (GSE17536) was performed to predict the function of AGXT2L1 in carcinomas of the digestive system. There were significant differences in PI metabolism and the synthesis of PIPs at the plasma membrane (Fig. 7A), between the two groups (AGXT2L1 low expression vs. high expression). To investigate the role of AGXT2L1 in autophagy, HCT116 and SW480 cells were transfected with AGXT2L1-siRNA and NC-siRNA. Expression levels of LC3, specifically LC3II, were increased in AGXT2L1-knockdown cells (Fig. 7B). In the immunofluorescence assay (Fig. 7C), LC3 was consistently expressed in a diffuse pattern in the control group, whereas in the AGXT2L1-knockdown group, the pattern of LC3 was changed from diffuse to punctate. These results provided preliminarily validation that AGXT2L1 
A

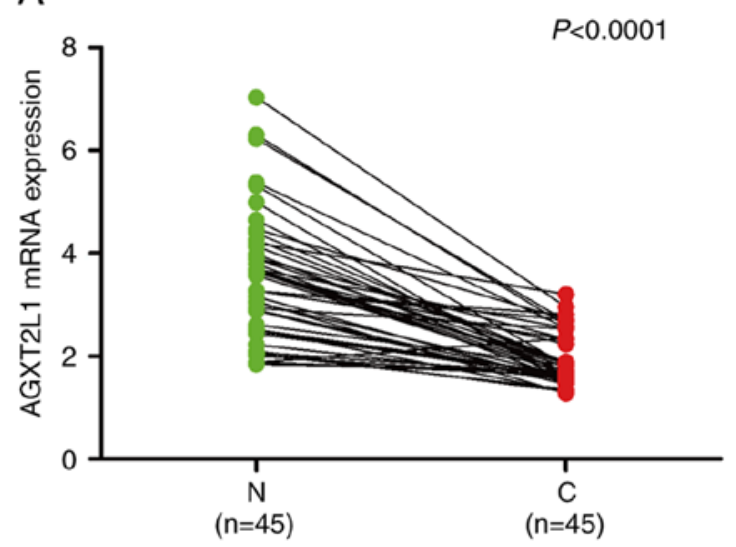

C

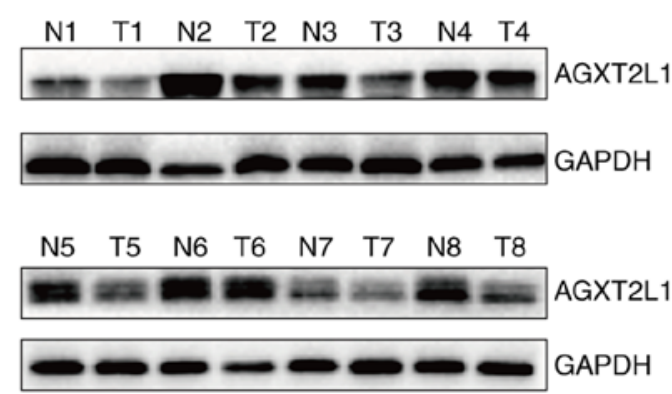

B
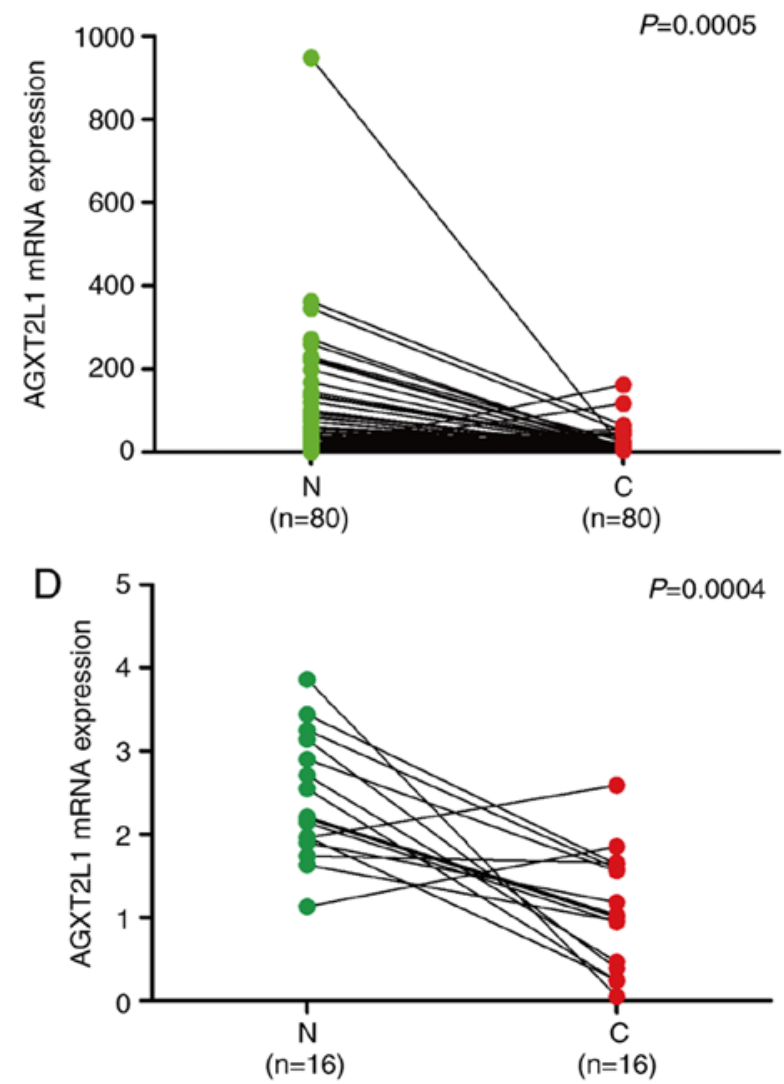

Figure 5. AGXT2L1 expression is reduced at the protein and mRNA levels in patients with GC. AGXT2L1 mRNA expression in adjacent normal gastric tissues and tumor tissues of patients with GC from two independent cohorts, (A) GSE63089 and (B) GSE27342 (both P<0.05). (C) AGXT2L1 protein levels determined by western blotting in paired GC tissues and normal gastric tissues. (D) AGXT2L1 mRNA expression in 16 matched GC tissues and normal tissues. GC, gastric cancer; AGXT2L1, alanine-glyoxylate aminotransferase 2-like 1; N, normal; C, cancer.
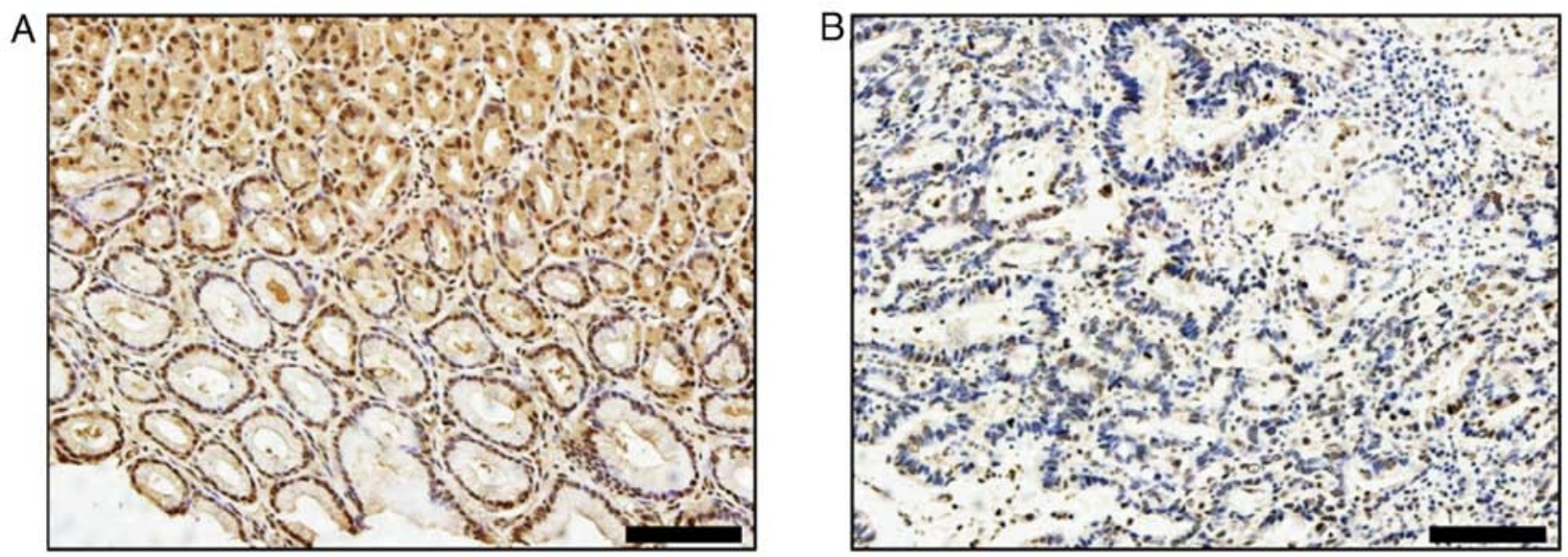

Figure 6. AGXT2L1 expression is decreased in PC. Representative immunohistochemistry images of (A) adjacent normal pancreatic tissue and (B) PC tissue. Scale bars, $100 \mu \mathrm{m}$. PC, pancreatic cancer; AGXT2L1, alanine-glyoxylate aminotransferase 2-like 1.

is involved in autophagy and it may modulate autophagy via phospholipid metabolism, thus contributing to cancer development.

\section{Discussion}

To the best of our knowledge, the present study is the first to examine the expression levels of AGXT2L1 in CRC, GC, PC and esophageal cancer. The limited sample size is a limitation of the present study, and it would be beneficial to include more samples in future studies to confirm the current findings. The findings of the present study were consistent with those of a previous study (5) on liver cancer, demonstrating that AGXT2L1 is significantly downregulated in cancerous tissues and may be a tumor suppressor; at least in carcinomas of the digestive system of colorectum, stomach and pancreas. 
A

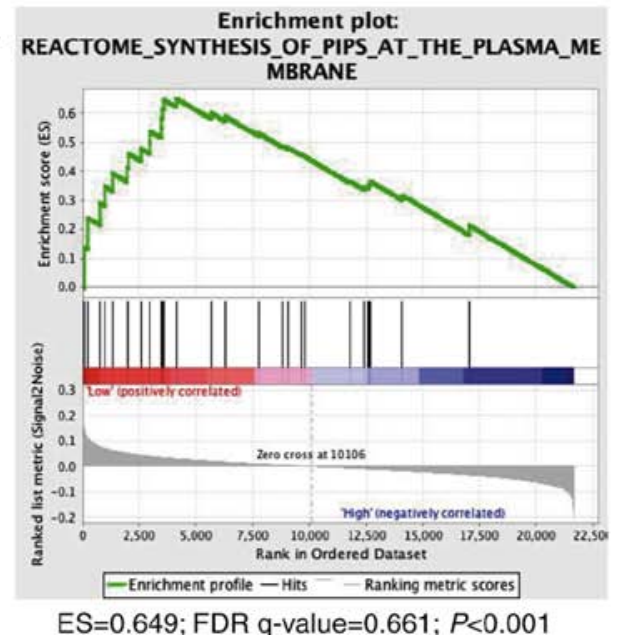

B

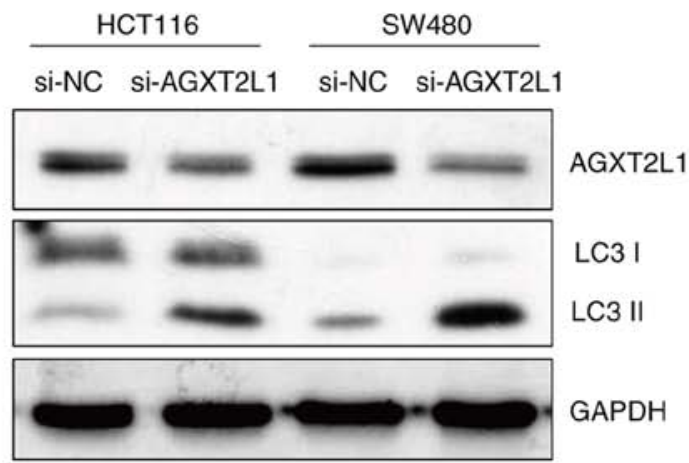

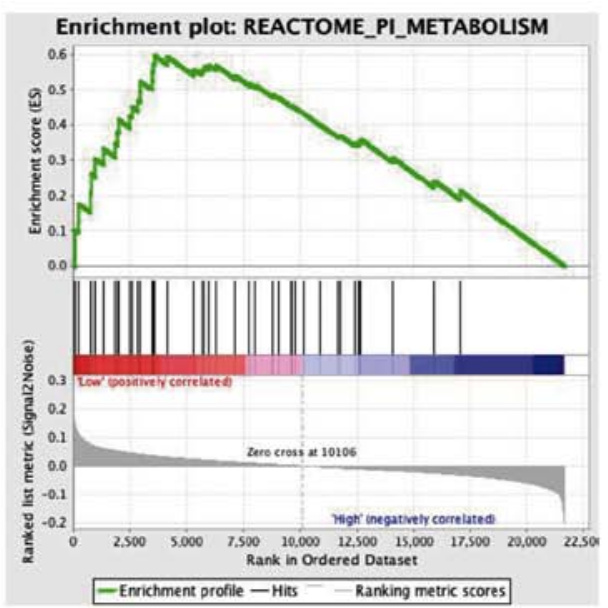

ES=0.597; FDR q-value $=0.645 ; P=0.0019$

C si-NC

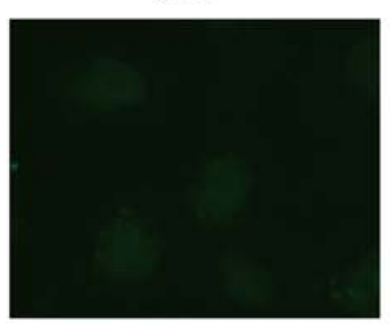

si-AGXT2L1

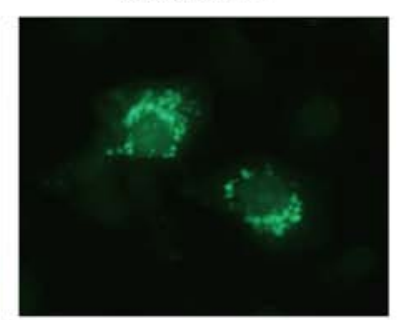

Figure 7. AGXT2L1 is associated with phosphatidylinositol metabolism and is involved in autophagy. (A) Gene set enrichment analysis of GSE17536 indicated that AGXT2L1 may regulate PI metabolism and PIPs biosynthesis in colorectal cancer. (B) AGXT2L1 expression was decreased by siRNA transfection in HCT116 and SW480 cells, whereas the expression of the autophagy marker LC3II was increased. (C) LC3 was detected by immunofluorescence (magnification, $\mathrm{x} 400$ ). In AGXT2L1 knockdown cells, the pattern of LC3 was altered from diffuse to punctate. AGXT2L1, alanine-glyoxylate aminotransferase 2-like 1; ES, enrichment score; FDR, false discovery rate; NC, negative control; PI, phosphatidylinositol; PIP, phosphatidylinositol phosphates; si-, small interfering RNA.

However, the underlying mechanism by which AGXT2L1 regulates tumorigenesis and cancer progression remains unclear, and several possible explanations are proposed here.

The present study demonstrated an association between AGXT2L1 and autophagy. The role of autophagy in the process of tumor development has always been considered as a double-edged sword (16). However, it is certain that autophagy can be activated to convert unnecessary molecules to essential substances to promote tumor growth in established cancer (17-19). For numerous years, the origin of the autophagosomal membranes and the regulation underlying autophagosome biogenesis aroused researchers' interests. It has first been suggested that the phagophore is derived from the endoplasmic reticulum (20). Subsequently, there have been various assumptions that the membrane might come from the Golgi, mitochondria, endosomes or the plasma membrane (20). Currently, it is widely accepted that autophagosomes are derived from endoplasmic reticulum membranes, which are strongly enriched in $\mathrm{PI}(3) \mathrm{P}(21,22)$. The present study demonstrated that AGXT2L1 may regulate PI metabolism and the synthesis of PIPs at the plasma membrane. PI is a membrane phospholipid that can be reversibly phosphorylated to generate several phosphoinositides, including phosphatidylinositol 3-phosphate $[\mathrm{PI}(3) \mathrm{P}](21,22)$. It has been reported that autophagosomes are derived from the endoplasmic reticulum membrane, which is strongly enriched in PI(3)P (22); thus, it was hypothesized that AGXT2L1 may regulate the process of autophagy. Therefore, it was speculated that AGXT2L1 may serve a role in the formation of the omegasome. On the other hand, PE is an indispensable component in the final step of autophagosome formation (23). It has been reported that a high level of PE can stimulate the conjugation of the ATG8/LC3-PE complex, which is necessary during the elongation and closure of the autophagic membrane $(9,24)$. The present study suggested that the downregulation of AGXT2L1 may result in excessive storage of PEtN and an increase in PE synthesis. In contrast to the mainstream view that PE may derive from multiple membrane sources, the present study suggested that the increased level of PE in autophagosomes may be due to the abnormal AGXT2L1 expression.

Compared with normal cells, cancer cells are relatively undifferentiated (25). Aberrant metabolism of phospholipids is a crucial characteristic during tumorigenesis and cancer progression (26). A number of studies have previously linked PE to cell differentiation (26-28). For example, lysophosphatidylethanolamine (LPE)-acyltransferase, which regulates the synthesis of PE from LPE, has been reported to modulate cardiac muscle differentiation (27). Lactamase $\beta$ is significantly upregulated in differentiated postmitotic muscle cells relative to its expression in undifferentiated, actively cycling 
cells and functions as a tumor suppressor by inhibiting phosphatidylserine decarboxylase, in turn blocking the synthesis of mitochondrial PE (26). As PEtN is the main precursor of PE, and AGXT2L1 irreversibly and strictly degrades PEtN, the downregulation of PEtN levels by AGXT2L1 may lead to inactivation of the synthesis of PE and subsequent differentiation.

Furthermore, the imbalance of the membrane PE/phosphatidylcholine ratio has a great impact on multiple cellular processes, such as lipogenesis, endoplasmic reticulum stress, mitochondrial respiration and oxidative capacity (29). PE and $\mathrm{PC}$ are the most abundant phospholipids in the cell membrane, and a decreased $\mathrm{PE} / \mathrm{PC}$ ratio leads to increased cell membrane permeability, resulting in the leakage of intracellular material that may trigger a series of inflammatory responses (30), which are stimulating factors in the process of tumorigenesis. All aforementioned factors are involved in cancer biology, and it is worth studying whether the downregulation of AGXT2L1 can take part in cancer progression via these mechanisms in the future.

\section{Acknowledgements}

The authors would like to thank Mr. Xia Hong from Hubei Key Laboratory of Digestive System, Renmin Hospital of Wuhan University (Wuhan, China) for his administrative support and excellent technical assistance in this work.

\section{Funding}

The present study was supported by National Natural Science Foundation of China (grant nos. 81672387 and 81703030).

\section{Availability of data and materials}

The datasets used and/or analyzed during the present study are available from the corresponding author on reasonable request.

\section{Authors' contributions}

QD and HY designed the experiments. YD and LW performed the experiments. YD and LW collected the tissues samples. YD and LW performed the statistical analyses. QD, LW and YD wrote the manuscript. HY and QD provided the funds and materials.

\section{Ethics approval and consent to participate}

The Ethics Committee of Renmin Hospital of Wuhan University approved the present study (approval no. WDRY2016-K033). Written informed consent was obtained from each patient prior to the study start.

\section{Patient consent for publication}

Not applicable.

\section{Competing interests}

The authors declare that they have no competing interests.

\section{References}

1. Torre LA, Bray F, Siegel RL, Ferlay J, Lortet-Tieulent J and Jemal A: Global cancer statistics, 2012. CA Cancer J Clin 65: 87-108, 2015

2. Veiga-da-Cunha M, Hadi F, Balligand T, Stroobant V and Van Schaftingen E: Molecular identification of hydroxylysine kinase and of ammoniophospholyases acting on 5-phosphohydroxy-L-lysine and phosphoethanolamine. J Biol Chem 287: 7246-7255, 2012.

3. Schiroli D, Cirrincione S, Donini S and Peracchi A: Strict reaction and substrate specificity of AGXT2L1, the human O-phosphoethanolamine phospho-lyase. IUBMB Life 65: 645-650, 2013.

4. Shao L and Vawter MP: Shared gene expression alterations in schizophrenia and bipolar disorder. Biol Psychiatry 64: 89-97, 2008.

5. Ding Q, Kang J, Dai J, Tang M, Wang Q, Zhang H, Guo W, Sun R and Yu H: AGXT2L1 is down-regulated in heptocellular carcinoma and associated with abnormal lipogenesis. J Clin Pathol 69: 215-220, 2016

6. Lai K, Killingsworth MC and Lee CS: The significance of autophagy in colorectal cancer pathogenesis and implications for therapy. J Clin Pathol 67: 854-858, 2014.

7. Saha S, Panigrahi DP, Patil S and Bhutia SK: Autophagy in health and disease: A comprehensive review. Biomed Pharmacother 104: 485-495, 2018.

8. PavlovicZandBakovicM:RegulationofPhosphatidylethanolamine Homeostasis - The Critical Role of CTP: Phosphoethanolamine Cytidylyltransferase (Pcyt2). Int J Mol Sci 14: 2529-2550, 2013.

9. Martens S, Nakamura S and Yoshimori T: Phospholipids in Autophagosome Formation and Fusion. J Mol Biol: Oct 27, 2016 (Epub ahead of print). doi: 10.1016/j.jmb.2016.10.029.

10. Ravikumar B, Sarkar S, Davies JE, Futter M, Garcia-Arencibia M, Green-Thompson ZW, Jimenez-Sanchez M, Korolchuk VI, Lichtenberg M, Luo S, et al: Regulation of mammalian autophagy in physiology and pathophysiology. Physiol Rev 90: 1383-1435, 2010.

11. Smith JJ, Deane NG, Wu F, Merchant NB, Zhang B, Jiang A, Lu P, Johnson JC, Schmidt C, Bailey CE, et al: Experimentally derived metastasis gene expression profile predicts recurrence and death in patients with colon cancer. Gastroenterology 138: 958-968, 2010

12. Zhang X, Ni Z, Duan Z, Xin Z, Wang H, Tan J, Wang G and Li F: Overexpression of E2F mRNAs associated with gastric cancer progression identified by the transcription factor and miRNA co-regulatory network analysis. PLoS One 10: e0116979, 2015

13. Cui J, Chen Y, Chou WC, Sun L, Chen L, Suo J, Ni Z, Zhang M, Kong X, Hoffman LL, et al: An integrated transcriptomic and computational analysis for biomarker identification in gastric cancer. Nucleic Acids Res 39: 1197-1207, 2011.

14. Cui J,Li F, Wang G, Fang X, Puett JD and Xu Y: Gene-expression signatures can distinguish gastric cancer grades and stages. PLoS One 6: e17819, 2011.

15. Livak KJ and Schmittgen TD: Analysis of relative gene expression data using real-time quantitative PCR and the 2(-Delta Delta C(T)) Method. Methods 25: 402-408, 2001.

16. 16. Gual P, Gilgenkrantz $\mathrm{H}$ and Lotersztajn S: Autophagy in chronic liver diseases: The two faces of Janus. Am J Physiol Cell Physiol 312: C263-C273, 2017.

17. Kowalik MA, Perra A, Ledda-Columbano GM, Ippolito G, Piacentini M, Columbano A and Falasca L: Induction of autophagy promotes the growth of early preneoplastic rat liver nodules. Oncotarget 7: 5788-5799, 2016

18. Sui X, Jin L, Huang X, Geng S, He C and Hu X: p53 signaling and autophagy in cancer: A revolutionary strategy could be developed for cancer treatment. Autophagy 7: 565-571, 2011.

19. Giatromanolaki A, Koukourakis MI, Harris AL, Polychronidis A, Gatter KC and Sivridis E: Prognostic relevance of light chain 3 (LC3A) autophagy patterns in colorectal adenocarcinomas. Clin Pathol 63: 867-872, 2010.

20. Tooze SA: Current views on the source of the autophagosome membrane. Essays Biochem 55: 29-38, 2013.

21. Hurley JH and Young LN: Mechanisms of Autophagy Initiation. Annu Rev Biochem 86: 225-244, 2017.

22. Lamb CA, Yoshimori T and Tooze SA: The autophagosome: Origins unknown, biogenesis complex. Nat Rev Mol Cell Biol 14: 759-774, 2013. 
23. Qiu Y, Zheng Y, Wu KP and Schulman BA: Insights into links between autophagy and the ubiquitin system from the structure of LC3B bound to the LIR motif from the E3 ligase NEDD4. Protein Sci 26: 1674-1680, 2017.

24. Rockenfeller P, Koska M, Pietrocola F, Minois N, Knittelfelder O, Sica V, Franz J, Carmona-Gutierrez D, Kroemer G and Madeo F: Phosphatidylethanolamine positively regulates autophagy and longevity. Cell Death Differ 22: 499-508, 2015.

25. Garcia H, Fleyshman D, Kolesnikova K, Safina A, Commane M, Paszkiewicz G, Omelian A, Morrison C and Gurova K: Expression of FACT in mammalian tissues suggests its role in maintaining of undifferentiated state of cells. Oncotarget 2: 783-796, 2011.

26. Keckesova Z, Donaher JL, De Cock J, Freinkman E, Lingrell S, Bachovchin DA, Bierie B, Tischler V, Noske A, Okondo MC, et al: LACTB is a tumour suppressor that modulates lipid metabolism and cell state. Nature 543: 681-686, 2017.

27. Fotheringham J, Xu FY, Nemer M, Kardami E, Choy PC and Hatch GM: Lysophosphatidylethanolamine acyltransferase activity is elevated during cardiac cell differentiation. Biochim Biophys Acta 1485: 1-10, 2000.
28. Wu Y, Chen K, Xing G, Li L, Ma B, Hu Z, Duan L and Liu X: Phospholipid remodeling is critical for stem cell pluripotency by facilitating mesenchymal-to-epithelial transition. Sci Adv 5: eaax 7525, 2019.

29. van der Veen JN, Kennelly JP, Wan S, Vance JE, Vance DE and Jacobs RL: The critical role of phosphatidylcholine and phosphatidylethanolamine metabolism in health and disease. Biochim Biophys Acta Biomembr 1859B: B1558-B1572, 2017.

30. Li Z, Agellon LB, Allen TM, Umeda M, Jewell L, Mason A and Vance DE: The ratio of phosphatidylcholine to phosphatidylethanolamine influences membrane integrity and steatohepatitis. Cell Metab 3: 321-331, 2006.

This work is licensed under a Creative Commons Attribution-NonCommercial-NoDerivatives 4.0 International (CC BY-NC-ND 4.0) License. 